\title{
Thyroid-Stimulating Hormone Values in Pregnancy: Cutoff Controversy Continues?
}

\author{
Suvarna Khadilkar ${ }^{1}$
}

Received: 23 August 2019 / Accepted: 23 August 2019 / Published online: 13 September 2019

(c) Federation of Obstetric \& Gynecological Societies of India 2019

\begin{abstract}
Thyroid disorders in pregnancy are important causes of adverse pregnancy outcome. So it is very pertinent that thyroid function is maintained in normal range during pregnancy. Serum thyroid-stimulating hormone (TSH) value is the best indicator for assessing and monitoring thyroid function. The increasing metabolic demands of pregnancy alter the thyroid physiology in early pregnancy; hence, it becomes necessary to define trimester-specific reference range. Several reports and guidelines have been published recommending varied TSH cutoffs in different studies. The most significant guidelines which created controversy about TSH cutoffs was that of American Thyroid Association (ATA) (Stagnaro-Green et al. in Thyroid 21:10811125,2011 ) followed by Endocrine Society clinical practice guideline (De Groot et al. in J Clin Endocrinol 97:2543-2565, 2012). Both these gave stricter TSH cutoffs as .1 to $2.5 \mathrm{mIU} / \mathrm{L}$ in first trimester, .2 to $3.0 \mathrm{mIU} / \mathrm{L}$ in second trimester and .3 to $3 \mathrm{mIU} / \mathrm{L}$ in third trimester. Subsequently many reports, meta-analysis and systematic reviews were published which recommended higher cutoffs. With due consideration, ATA revised the guidelines in 2017, recommending the upper cutoff limit $.5 \mathrm{mIU} / \mathrm{L}$ less than the preconception TSH value or as $4.0 \mathrm{mIU} / \mathrm{L}$ when local population-specific reference range is not available (Alexander et al. Thyroid 27(3):315-389, 2017). The controversy is not yet completely resolved specially regarding management of subclinical hypothyroidism. This editorial addresses this ongoing controversy.
\end{abstract}

Keywords Indian reference range $\cdot$ Normative data $\cdot$ TSH $\cdot$ Thyroid-stimulating hormone $\cdot$ Pregnancy $\cdot$ Thyroid function $\cdot$ Subclinical hypothyroidism $\cdot$ Pregnancy outcome $\cdot$ Recurrent pregnancy loss

\section{Introduction}

Thyroid disorders in pregnancy are associated with poor pregnancy outcome. Both hypothyroidism and hyperthyroidism can lead to adverse obstetric outcome. The prevalence of hypothyroidism reported in India is $12 \%$, whereas hyperthyroidism is seen in $1.25 \%$ in pregnant women [1] so

Prof. Suvarna Satish Khadilkar MD DGO FICOG, CIMP, Diploma in Endocrinology (UK) is Editor in Chief of Journal of Obstetrics and Gynecology of India, and Treasurer, FOGSI; she is Professor and Head, and Consultant Gyne-Endocrinologist, Bombay Hospital and Medical Research Centre, Mumbai, Former Professor and Head, Dept of Ob-Gyn, RCSM, Government Medical College, Maharashtra and Associate Professor and Unit Chief Grant Medical College and Cama and Albless Hospital, Mumbai.

Suvarna Khadilkar

suvarnakhadilkar2@gmail.com

1 Bombay Hospital and Medical Research Centre, Mumbai, India universal screening of TSH in pregnancy is recommended. Severe uncontrolled hypothyroidism is seldom associated with pregnancy as patients probably do not get pregnant with severe disease. However, patients with mild hypothyroidism or subclinical hypothyroidism may conceive and their minor thyroid abnormality may go unnoticed. Uncontrolled thyrotoxicosis in pregnancy is associated with a higher risk of pregnancy complications like preeclampsia, intrauterine growth restriction (IUGR). Cardiac failure may ensue due to excess thyroid secretion as a demand of pregnancy, superimposed by hyper-dynamic circulation of pregnancy. Issue of subclinical hyperthyroidism does not cause significant pregnancy complications.

So it is very important that we maintain TSH values within normal gestation-specific reference range. Last several years normative data for TSH values in pregnancy are being defined and redefined and have led to controversies!

The literature has varied reports, guidelines and recommendations giving different TSH cutoff values. The guidelines are revised essentially to optimize the best cutoff value 
to achieve the best maternal and perinatal outcome and minimize the complications. However, they have created some controversies in clinical management.

This editorial takes an account of the current controversies regarding TSH cutoffs in pregnancy focusing more on subclinical hypothyroidism.

\section{Thyroid Physiology in Pregnancy}

The effect of pregnancy on thyroid physiology is well understood and documented. The salient features are given in Table 1. Because of increased metabolic demand of pregnancy, there is increased basal metabolic rate, increased secretion of thyroid hormone due to action of human chorionic gonadotrophin (HCG) and human chorionic thyrotropin (HCT) which share their molecular structure, as well as receptor cross-reactivity with TSH. HCG bears $1 / 4000$ th potency to stimulate thyroid activity compared with TSH [2], but very high levels of HCG in normal pregnancy are enough to bring about a state of physiological thyrotoxicosis, and T4 levels tend to be higher in all conditions with excess HCG titers for, e.g., vesicular mole, multiple pregnancy, etc.

Transfer of thyroid hormone across the placenta is limited; however, even a small amount of transfer will provide protection, especially to the fetal brain.

\section{Normative Data of TSH Values with Trimester-Specific Reference Range}

When thyroid physiology undergoes alteration in early pregnancy by way of increasing demand and increased secretion of thyroid hormone, the cutoffs used in non-pregnant women will not hold true in pregnancy. In early pregnancy, lower cutoffs are considered normal. The ATA [3] recommends that reference range should be calculated locally, and if not available 0.4 to 4.5 should be considered normal range in non-pregnant adults. This cutoff should be maintained at 0.1 to 2.5 in the first trimester and at 0.2 to 3.0 in the second trimester and 0.3 to 3 in the third trimester as per ATA, 2011 [3] as well as endocrinology society guidelines, 2012 [4].

However, on reviewing literature reports published thereafter, ATA revised their recommendations in 2017 [5]. They recommended that the first trimester upper normal limit cutoff should be obtained by deducting ' $0.5 \mathrm{mIU} / \mathrm{L}$ ' from prepregnancy TSH value. But if it is not known then ' $4.0 \mathrm{mIU} / \mathrm{L}$ ' should be taken as upper limit of normal cutoff.

It is recommended that each laboratory should have their gender, ethnicity, season, circadian and assay-specific reference ranges set. However, one must remember having TSH values within normal range of laboratory does not necessarily mean that the thyroid function is normal. We must understand that reference range for individual may be abnormal which can only be set for each individual by taking several samples and that will give clear idea about normalcy for that individual. But it is impractical to measure individual reference range for all. The individual reference range for serum TSH is approximately half the width of laboratory reference range. Thus, some individuals in the upper and lower parts of the normal range will have TSH outside their individual reference range. The literature review shows that authors across all the studies choose variable percentile cutoffs. Some choose 5th and 95th percentile cutoffs, while others choose 2.5th and 97.5th percentiles.

Table 1 Thyroid physiology in pregnancy

\begin{tabular}{|c|c|}
\hline Maternal physiology & Fetal and neonatal physiology \\
\hline High basal metabolic rate (BMR) & $\begin{array}{l}\text { TSH and T4 appear in the fetus at } 10-13 \text { weeks and } \\
\text { rise abruptly at } 20 \text { weeks }\end{array}$ \\
\hline Increased metabolic demands & $\begin{array}{l}\text { T4 rises rapidly and exceeds maternal values at } \\
\text { term but very low T3 levels }\end{array}$ \\
\hline Increased iodine uptake & Reverse T3 (RT3) levels exceed normal adult levels \\
\hline Increased size of gland-hyperplasia and hypervascularity & TSH peaks at $30 \mathrm{~min}$ of age \\
\hline Iodide clearance increased & $\mathrm{T} 3$ peaks at $24 \mathrm{~h}$ \\
\hline Iodide loss to fetus reduces iodide levels & $\mathrm{T} 4$ peaks at $24-48 \mathrm{~h}$ \\
\hline Transfer of TSH, T3 and T4 across placenta is limited but sufficient for neuroprotection & High RT3 reaches normal values by 2 weeks \\
\hline \multicolumn{2}{|l|}{ HCG peak at 10 weeks corresponding to nadir of TSH } \\
\hline \multicolumn{2}{|l|}{ Marked increase in thyroid binding globulin (TBG) peak at 15 weeks } \\
\hline \multicolumn{2}{|l|}{ Increased activity of thyroid gland due to direct stimulation by HCG and HCT } \\
\hline \multicolumn{2}{|l|}{ Decreased free $\mathrm{T} 3$ and free $\mathrm{T} 4$ levels but within normal range } \\
\hline $\begin{array}{l}\text { Hyperemesis gravidarum and transient hyperthyroidism due to high specific subgroup of } \\
\text { HCG with reduced sialic acid content }\end{array}$ & \\
\hline
\end{tabular}




\section{Is There Any Impact of Subclinical Hyperthyroidism on pregnancy?}

Subclinical excess of thyroid secretion has not been associated with adverse pregnancy outcomes. Therefore, a maternal TSH concentration that is low but detectable is not likely to be clinically significant. Revised ATA guidelines 2017 [5] recommend lower limit of TSH cutoffs as $0.1 \mathrm{mIU} / \mathrm{L}$ in the first trimester, $0.2 \mathrm{mIU} / \mathrm{L}$ in the second trimester and $0.3 \mathrm{mIU} / \mathrm{L}$ in the third trimester.

\section{What is Subclinical Hypothyroidism?}

Subclinical hypothyroidism is an abnormally high thyroidstimulating hormone (TSH) level with normal free thyroxine level without symptoms of hypothyroidism [6]. After one abnormal TSH value, the TSH should be repeated after 6 weeks. If TSH persistently shows values above the normal limit with normal FT4, it confirms the biochemical diagnosis of subclinical hypothyroidism ( $\mathrm{SCH})$. The prevalence of $\mathrm{SCH}$ is $4-8 \%$ in non-pregnant women. For non-pregnant women, the TSH upper normal limit is typically 4.2 to $4.5 \mathrm{mIU} / \mathrm{L}$ [7].

Serum TSH is very sensitive to minor changes in thyroid hormone concentrations in serum. Hence, an abnormal TSH but $\mathrm{T} 4$ and $\mathrm{T} 3$ within laboratory reference ranges is probably always a sign that $\mathrm{T} 4$ and $\mathrm{T} 3$ are outside the individual reference range and thus indicate abnormal thyroid function in the individual. For this reason, $\mathrm{SCH}$ requires action when symptoms are present. The controversy exists regarding at what cutoff of TSH should LT4 therapy begin.

\section{What is Gestational Hypothyroidism?}

On the literature review, only one article referring to the term 'gestational hypothyroidism' was found [8]. In preconceptional stage, those women who have TSH value within normal or high-normal range closer to $4.5 \mathrm{mU} / \mathrm{L}$, they are likely to have poor reserve of thyroid function. If such women conceive, the thyroid is unable to cope up with increasing metabolic demand of pregnancy, thus pregnancy acts as a thyroid challenge test, and a state of hypothyroidism ensues. This may be referred to as gestational hypothyroidism. The LT4 therapy may have to be initiated during pregnancy but may not be required after pregnancy, as thyroid function may return to normal. The pregnancy outcome is likely to be adverse if timely treatment is not started. Perhaps dysfunction of tissues such as the endometrium and the corpus luteum may be responsible for increased abortion rate. Subclinical hypothyroidism during pregnancy has been reported to be associated with adverse pregnancy outcomes and impaired fetal neurodevelopment in addition to increased rate of abortion.

\section{Is 2.5 Very Low Cutoff for Normal TSH in First Trimester?}

The American Thyroid Association (ATA) guideline 2011 [3] recommended the trimester-specific criteria of TSH concentration for the diagnosis of subclinical hypothyroidism. They defined reference ranges for first trimester, 0.1-2.5 mIU/L; second trimester, $0.2-3.0 \mathrm{mIU} / \mathrm{L}$; and third trimester, 0.3-3.0 mIU/L. They recommended these strict criteria for entire first trimester, but normal reference range of TSH concentrations was derived from women at 9 to 12 weeks of gestation, and many authors considered those cutoffs too low.

ATA revised their recommendation in 2017 and recommended to consider a cutoff value 0.5 lower than the prepregnancy level [5]. It is important that population specific ranges are used otherwise different cutoffs will lead to different prevalence rates of thyroid disorders in the same population. This is likely to lead to over diagnosis and over treatment. However TPO antibody positive women may not have favorable pregnancy outcome with cutoff values higher than 2.5 .

\section{Indian Scenario}

One of the early Indian reports on reference ranges for TSH states values of $0.6-5.0 \mathrm{mIU} / \mathrm{L}$ in the first, $0.44-5.78 \mathrm{mIU} / \mathrm{L}$ in the second and $0.74-5.7 \mathrm{mIU} / \mathrm{L}$ in the third trimester. They used 5th to 95 th percentile as normal reference range [9].

Recent Indian report states that cutoff of $4.0 \mathrm{mIU} / \mathrm{L}$ as per revised ATA, 2017, may be too high for our patients, and they suggested to use an intermediate cutoff of 3.00 rather than $4.0 \mathrm{mIU} / \mathrm{L}[10]$.

A systematic review took into account pooled data from selected eight Indian studies, and they recommended that TSH cutoffs of up to 5-6 mIU/L similar to prepregnancy stage should be used even in the first trimester of pregnancy. Strict criteria of $2.5 \mathrm{mIU} / \mathrm{L}$ as advised by ATA 2011 should be discouraged [11].

Current evidence is conflicting, and no uniform recommendation can be inferred from these reports. I believe that the reasons for these conflicting reports are multifold, like wide variations in study designs, no uniformity in exclusion criteria, different aims, use of different cutoffs of TSH percentile values and lack of data on TPO antibody statuses in many studies. Many of them have not followed all the 
women longitudinally and have not studied their pregnancy outcome. Some have cutoffs of 5 th to 95 th percentile values while others have 2.5 to 97.5 percentiles values as normal ranges, but we must understand that these percentile cutoffs give statistical probability of normal ranges. To confirm that these are normal, we must correlate these values with pregnancy outcomes of all these women. Therefore, there is a need for well-designed local population-based study which is cross-sectional as well as longitudinal to obtain the local trimester specific reference ranges of TSH, and also to study of the maternal and neonatal outcome of the same data set.

\section{What should be the Recommendations for Women Planning to Conceive to Achieve Best Outcome?}

Despite agreement for TSH screening in infertile women, there are insufficient data upon which recommendations can be based for treatment thresholds and optimal TSH goals during pregnancy.

Preconceptional euthyroid status: If women have TSH values in high-normal range of TSH, i.e., $2.5-4.5 \mathrm{mIU} / \mathrm{L}$, their thyroid reserve may be poor and they may not exhibit values $0.5 \mathrm{mIU} / \mathrm{L}$ lower than prepregnancy in the first trimester as is considered a normal thyroid function in pregnancy. LT4 treatment to bring TSH down may be justified in such borderline normal cases, in women with TPO antibodypositive status, and a history of recurrent pregnancy loss. Women having low-normal TSH values, may not require any action.

Preconceptional hypothyroidism status: Patient should be instructed to report as soon as pregnancy test is detected to be positive, for LT4 dose adjustment. The dose of LT4 should be immediately increased by $25-30 \%$. One should aim at monitoring and maintaining TSH in pregnancy-specific range by serial monthly TSH measurements.

Preconceptionally known SCH status: If woman is undergoing artificial reproductive techniques, TSH should be brought to less than $4.0 \mathrm{mIU} / \mathrm{L}$ with LT4 treatment as LT4 treatment has been associated with better reproductive outcomes in these women [12].

One study reported no difference in maternal clinical outcomes at TSH range of both $\geq 2.5-4.5 \mathrm{mIU} / \mathrm{L}$ and $\geq 4.5 \mathrm{mIU} / \mathrm{L}$. So question arises why should we treat women having TSH more than 4.5? It may be justified to offer LT4 treatment to these women undergoing ART procedures as risk of complications of LT4 therapy is very low [13].

ATA 2017 [5] recommends that all patients seeking pregnancy, or newly pregnant, should undergo clinical evaluation and assessment of serum TSH and TPO antibody status if risk factors are present like age more than 30, high BMI, infertility, bad obstetric history, diabetes mellitus, positive family history of thyroid disorders and last but not the least the history of prior thyroid disease or surgery.

TSH cutoff value for gestational SCH screening was reported to be $4.7 \mathrm{mIU} / \mathrm{L}$ in one study [14].

Authors observed that with $2.5 \mathrm{mIU} / \mathrm{L}$ TSH cutoff point, the prevalence of SCH rises to 37\%. Applying the ATA 2017 recommended cutoff point of $4 \mathrm{mIU} / \mathrm{L}$, the prevalence of $\mathrm{SCH}$ is $9.6 \%$. The prevalence of SCH drops to $5 \%$ when their own derived cutoff value of 4.7 was used [14].

For women who are first time diagnosed with $\mathrm{SCH}$ during pregnancy, a low dose of $50 \mathrm{mg}$ of LT4 can be started and titrated as necessary [5].

\section{Management of SCH in Pregnancy as per TPO Status (ATA) [5]}

Levothyroxine therapy is recommended for:

- TPO antibody-positive women with a TSH greater than the pregnancy-specific reference range

- TPO antibody-negative women with a TSH greater than $10.0 \mathrm{mU} / \mathrm{L}$.

Levothyroxine therapy may be considered for:

- TPO antibody-positive women with TSH concentrations $>2.5 \mathrm{mU} / \mathrm{L}$ and below the upper limit of the pregnancy-specific reference range.

- TPO antibody-negative women with TSH concentrations greater than the pregnancy-specific reference range and below $10.0 \mathrm{mU} / \mathrm{L}$.

Levothyroxine therapy is not recommended for:

- TPO antibody-negative women with a normal TSH (TSH within the pregnancy-specific reference range, or $<4.0 \mathrm{mU} / \mathrm{L}$ if unavailable).

\section{Management of the TPO antibody-positive euthyroid} women:

Euthyroid women with TSH $<2.5 \mathrm{mIU} / \mathrm{L}$ and TPO-positive status currently are not recommended for LT4 treatment. Despite a significant statistical correlation of higher rate of spontaneous abortion in women with positive thyroid peroxidase antibodies (TPO) in euthyroid women, LT4 therapy is not yet recommended. 
Negro et al. [15] compared 14 maternal and neonatal adverse outcomes in 245 thyroid peroxidase positive, euthyroid women ( $\mathrm{TSH}<2.5 \mathrm{mIU} / \mathrm{L}$ ) in the first trimester to 3348 women who were euthyroid and thyroid peroxidase negative in the first trimester [15]. They compared parameters such as hypertension, preeclampsia, gestational diabetes, cesarean section, preterm labor, respiratory distress, NICU admission, low-birth weight, preterm delivery, low apgar scores, neonatal death and other complications. They found that all the 14 parameters were significantly adversely affected in TPO-positive women.

One Indian study [16] reported that TPO antibody-positive euthyroid women had 4-5 times higher incidence of miscarriage and LBW irrespective of their gestational age as compared to TPO-negative euthyroid women. However, they did not find any correlation with the other complication as found in the study by Negro et al. [15].

The overall evidence is conclusive neither to recommend a routine screening for thyroid autoantibodies nor to recommend empirical LT4 therapy [17].

\section{Conclusion}

Review of various reports suggests that stricter criteria of 2.5 cutoff may be considered too low. Many women would be unnecessarily diagnosed as $\mathrm{SCH}$ and may be subjected to therapeutic burden of LT4 treatment. We must try to get normative data and reference range specific to local population and obtain entire range of percentile values [14, 18], study the pregnancy outcome prospectively and then recommend normal reference range as per outcome. It is always beneficial to use ethnicity-specific data; hence, we must use Indian reference ranges. I am sure the Indian reference range for TSH cutoffs calculated by well-designed prospective longitudinal study will go a long way in improving pregnancy outcome and reducing diagnostic and therapeutic pitfalls of thyroid disorders in pregnancy.

Until definitive evidence is available regarding normative data for TSH cutoff controversy continues...

\section{References}

1. Ajmani SN, Aggarwal D, Bhatia P, et al. Prevalence of overt and subclinical thyroid dysfunction among pregnant women and its effect on maternal and fetal outcome. J Obstet Gynecol India. 2014;64(2):105.

2. Fritz MA, Speroff L. Reproduction and thyroid, chapter 20, Clinical gynecologic endocrinology and infertility. 8th ed. Wolters Kluwer Health Lippincot Williams and Wilkins; 2010. pp. 340-2.
3. Stagnaro-Green A, Abalovich M, Alexander E, et al. Guidelines of the American Thyroid Association for the diagnosis and management of thyroid disease during pregnancy and postpartum. Thyroid. 2011;21:1081-125.

4. De Groot L, Abalovich M, Alexander EK, et al. Management of thyroid dysfunction during pregnancy and postpartum: an endocrine society clinical practice guideline. J Clin Endocrinol Metab. 2012;97:2543-65.

5. Alexander EK, Pearce EN, Brent GA, et al. 2017 Guidelines of the American Thyroid Association for the diagnosis and management of thyroid disease during pregnancy and the postpartum. Thyroid. 2017;27(3):315-89.

6. Lee YJ, Kim CH, Kwack JY et al. Subclinical hypothyroidism diagnosed by thyrotropin-releasing hormone stimulation test in infertile women with basal thyroid-stimulating hormone levels of 2.5 to $5.0 \mathrm{mIU} / \mathrm{L}$. Obstet Gynecol Sci. 2014;57:507-512.

7. Maraka S, Singh Ospina NM, Mastorakos G, et al. Subclinical hypothyroidism in women planning conception and during pregnancy: who should be treated and how? J Endocr Soc. 2018;2(6):533-46.

8. Hammond KR, Cataldo NA, Hubbard JA, et al. Gestational hypothyroidism: development of mild hypothyroidism in early pregnancy in previously euthyroid women. Fertil Steril. 2015;103(6):1532-6.

9. Marwaha RK, Chopra S, Gopalakrishnan S, et al. Establishment of reference range for thyroid hormones in normal pregnant Indian women. BJOG. 2008;115:602-6.

10. Kalra S, Agarwal S, Aggarwal R, et al. Trimester-specific thyroidstimulating hormone: an Indian perspective. Indian J Endocrinol Metab. 2018;22(1):1.

11. Kannan S, Mahadevan S, Sigamani A. A systematic review on normative values of trimester-specific thyroid function tests in Indian women. Indian J Endocr Metab. 2018;22:7-12.

12. Maraka S, Singh ON, Mastorakos G, et al. Subclinical hypothyroidism in women planning conception and during pregnancy: who should be treated and how? J Endocr Soc. 2018;2(6):533.

13. Reh A, Grifo J, Danoff A. What is a normal thyroid-stimulating hormone (TSH) level? Effects of stricter TSH thresholds on pregnancy outcomes after in vitro fertilization. Fertil Steril. 2010;94(7):2920-2.

14. Lara MC, Sánchez ÁV, Solano CC, Pazos ES, Álvarez MI, Macías CG, Ortega CA, Corral LJ, Alba JJ. Hypothyroidism screening during first trimester of pregnancy. BMC Pregnancy Childbirth. 2017;17(1):438.

15. Negro R, Schwartz A, Gismondi R, et al. Thyroid antibody positivity in the first trimester of pregnancy is associated with negative pregnancy outcomes. J Clin Endocrinol Metab. 2011;96(6):E920-24.

16. Meena A, Nagar P. Pregnancy outcome in euthyroid women with anti-thyroid peroxidase antibodies. J Obstet Gynecol India. 2016;66(3):160-5.

17. Mehran L, Tohidi M, Sarvghadi F, et al. Management of thyroid peroxidase antibody euthyroid women in pregnancy: comparison of the American Thyroid Association and the Endocrine Society Guidelines. J Thyroid Res. 2013; 2013, Article ID 542692. https ://doi.org/10.1155/2013/542692.

18. Medici M, Korevaar TI, Visser WE, et al. Thyroid function in pregnancy: what is normal? Clin Chem. 2015;61(5):704-13.

Publisher's Note Springer Nature remains neutral with regard to jurisdictional claims in published maps and institutional affiliations. 


\section{About the Author}

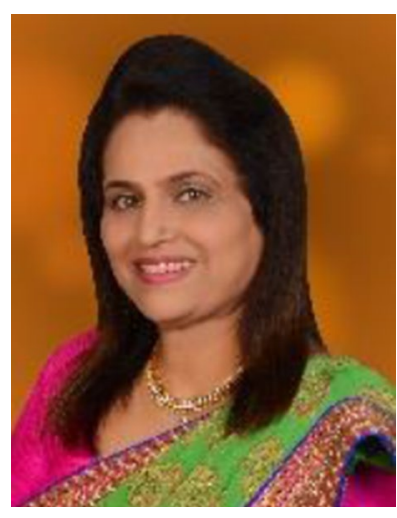

Dr. Suvarna Khadilkar is the editor in chief of this journal. She is currently Professor and head of the department of Obstetrics and Gynecology and Consultant Gyne-Endocrinologist, Bombay Hospital Institute of Medical Sciences and Medical Research Centre, Mumbai. In the past, she worked as an Associate Professor and Unit Chief at JJ Group of Hospitals and Grant Medical College [GMC], Mumbai, and thereafter in the capacity of the Professor and Head in Department of Obstetrics and Gynecology, Government Medical College, Kolhapur, Maharashtra. She has over 30 years of teaching experience as an undergraduate and postgraduate teacher and examiner, Mumbai University and Maharashtra
University of Health Sciences. Pursuing her interest in endocrinology, she acquired Diploma in Endocrinology from the prestigious University of South Wales, UK, and has been appointed as a recognized teacher in endocrinology in University of South Wales. She is currently the treasurer of FOGSI, treasurer of Mumbai Ob-Gyn Society, and treasurer of Association of Medical Women in India [AMWI]. She has held many prestigious positions like President of Indian Menopause Society, Chairperson of Reproductive Endocrinology Committee of FOGSI, President of AMWI, Mumbai. She has published six textbooks and more than 70 articles at national and international levels. She is recipient of 30 awards for her research work including the Young Scientist Award. 